\title{
KONGRESSKALENDER
}

\section{März 2018}

07.-09.03.2018, Lippstadt-Eickelborn 33. Eickelborner Fachtagung zu Fragen der Forensischen Psychiatrie

Dr. N. Saimeh

Kontaktadresse: Michaela.Riepe@

wkp-lwl.org

Information: www.lwl-forensik-lippstadt.de

16.03.2018, Tübingen

Forensisch-Psychiatrisches Gespräch

2018: Grenzen und Möglichkeiten

Forensischer Ambulanz

Psychiatrische Universitätsklinik, Hörsaal UKPP

Information: christel.weimer@med.

uni-tuebingen.de

\section{April 2018}

09.-11.04.2018, Potsdam-Griebnitzsee Begutachtungskurs Forensische Psychiatrie: Schwerpunkt Kriminalprognose Dr. F. Wendt, Prof. Dr. H.-L. Kröber

Zentrum für Forensisch- Psychiatrische Begutachtung

Information: www.zfpb.de; kroeber@zfpb.de

\section{Mai 2018}

11.05.2018, Dresden

23. Dresdner Frühjahrstagung für Forensische Psychiatrie

Dr. Lange, Prof. Dr. Bauer, Dr. Lammel, Dr. Sutarski

Information: www.forensik-dresden.de

30.05.-01.06.2018, Zürich, Schweiz 10. Internationales Symposium Forensische Psychiatrie

World Trade Center

Prof. Endrass, Dr. Rossegger, Prof. Urbaniok

Information: www.forensiktagung.ch

\section{Juni 2018}

12.06.-14.06.2018, Antwerpen, Belgien 18th Annual IAFMHS Conference Forensic Mental Health Services Information: www.iafmhs.org

12.-14.06.2018, Stockholm, Schweden The Stockholm Criminology Symposium Swedish National Council of Crime Prevention

City Conference Center, Stockholm Information: www.criminologysymposium.com

15.06.2018, Berlin-Dahlem

22. Berliner Junitagung für Forensische Psychiatrie und Psychologie Forensische Psychiatrie Psychologie Berlin e. V. (FPPB) \& Institut für Forensische Psychiatrie der Charité

Information: www.forensik-berlin.de; www.zfpb.de (Veranstaltungen)

26.-29.06.2018, Turku, Finland European Association of Psychology and Law (EAPL) 28 ${ }^{\text {th }}$ Annual Conference Uses and limits of psychological expertise in legal processes

Information: http://eapl2018.fi/

\section{August 2018}

28.-31.08.2018, Vilnius, Litauen

15th Conference International Association for the Treatment of Sexual Offenders (IATSO)

Information: www.iatso.org

29.08-01.09.2018, Sarajevo, Bosnien European Association of Criminology 18th Annual ESC Conference Information: http://www.esc-eurocrim.org/

\section{Oktober 2018}

Oktober 2018, München

33. Herbsttagung für Forensische Psychiatrie

Abteilung für Forensische Psychiatrie der Universität München

Information: www.forensik-muenchen.de

17.-20.10.2018, Vancouver, BC, Canada Association for the Treatment of Sexual Abusers (ATSA)

Hyatt Regency Vancouver

Information: http://www.atsa.com/futureatsa-conferences

23.-25.10.2018, Bergen, Norwegen $5^{\text {th }}$ Bergen International Conference on Forensic Psychiatry

Neurocognitive Disorders Across the Lifespan

Information: www.bergenconference.no

25.-28.10.2018, Austin, Texas (USA)

American Academy of Psychiatry and the Law

$4^{\text {th }}$ Annual Meeting (with APA)

JW Marriott Austin

Information: www.AAPL.org

\section{November 2018}

14.-17.11.2018 Atlanta, GA (USA) American Society of Criminology Annual Meeting Institutions, Cultures, and Crime. Atlanta Marriott Marquis Information: http://www.asc41.com/ annualmeeting.htm

Hinweise für den Kongresskalender bitte an: mail@hlkroeber.de 